\title{
Variantes del español colombiano y su efecto en la enseñanza del español como lengua extranjera. Marco Sociolingüístico*
}

\author{
RAFAEL AREIZA LONDOÑO* \\ rafaelareizalondono@gmail.com \\ MARÍA DEL PILAR FLÓREZ OSPINA" \\ pilar.florez@caroycuervo.gov.co
}

Forma de citar este artículo: Areiza Londoño, R., \& Flórez Ospina, M. P. (2016). Variantes del español colombiano y su efecto en la enseñanza del español como lengua extranjera. Marco sociolingüístico. Cuadernos de Lingüística Hispánica, (27), 79-107.

* $\quad$ Este artículo de investigación, enmarcado dentro del macroproyecto de investigación "Caracterización de las variedades del español de Colombia y su efecto en los estudios del Español como Lengua Extranjera (ele)", financiado por el Instituto Caro y Cuervo, Colombia.

** Magíster en Linguística y Español de la Universidad del Valle, Colombia; miembro del grupo de investigación "Estudios del lenguaje y la educación" y catedrático de la Universidad de La Salle, Bogotá, Colombia.

*** Magíster en Lingüística Española del Instituto Caro y Cuervo, Colombia, coordinadora e investigadora de la línea de Español como Lengua Extranjera, Segunda Lengua y Bilingüismo del mismo instituto y catedrática de la Universidad de La Salle, Colombia. 


\title{
Resumen
}

En la construcción de la lengua como sistema interactivo se requieren dos grandes entidades: un sistema formal y la sociedad con todas sus cargas ideológicas y culturales, que le dan identidad al sistema y lo caracterizan en sus particularidades simbólicas. Este macrosistema social y lingüístico presenta variantes en los distintos contextos donde se pone en escena y, por su carácter idiosincrático, expresa un modo de actuar y estar en el mundo que debe ser reconocido por todas las comunidades. La enseñanza del Español como Lengua Extranjera (elE) debe estar acompañada del conocimiento de esas variantes sociolingüísticas, sin prescindencia de la variante estándar, para buscar la integración del aprendiente al contexto, sin el riesgo de rechazar o ser rechazado por el medio donde le toque desenvolverse. El presente artículo tiene dos objetivos muy concretos: mostrar algunas de las variantes que tiene el español en Colombia ubicándolas como expresión de grupos culturales del país que merecen ser reconocidos con sus características, y valorar las variantes como objeto de trabajo dentro de contextos académicos reivindicando su importancia en el contexto nacional.

Palabras clave: modo de vida, variante, sistema, cognición social, mercado linguístico, simbolización, redes sociales, aprendiente de ele.

\section{Colombian Spanish variations and their effect on the teaching of Spanish as a Foreign Language. A sociolinguistic framework.}

\begin{abstract}
For the construction of a language as an interactive system, two great entities are required: a formal system and society, with all the ideological and cultural meaning that give identity to that system and characterize it in its distinctive symbolic features. This social and linguistic macrosystem presents variations in the different contexts where it is activated and, due to its idiosyncratic nature, it expresses a way of acting and being in the world that must be recognized by all communities. The teaching of Spanish as a Foreign Language (SFL) must be accompanied by the knowledge of its sociolinguistic variations, without forgetting the standard variation, in order that the learner assimilates the context, avoiding the risk of rejecting or being rejected by the enviroment in which he will use this language. This article has two very specific objectives: to show some of the variations of Colombian
\end{abstract}


Spanish, locating them as expressions of different cultural groups of the country that may be recognized by distinctive features, and to value these variations as an object of study within academic contexts, reclaiming their importance in the national context.

Key Words: way of life, variation, system, social cognition, linguistic market, symbolization, social media, SFL learner.

\section{Variantes de l'espagnol colombien et son effet dans l'enseignement de l'espagnol langue étrangère. Cadre sociolinguistique.}

\section{Résumé}

Dans la construction de la langue en tant que système interactif, on requiert deux grandes entités: un système formel et la société avec toutes ses charges idéologiques et culturelles, qui donnent de l'identité au système et le caractérisent dans ses particularités symboliques. Ce macro-système social et linguistique présente des variantes dans les divers contextes où on le met en scène et, pour son caractère idiosyncratique, il exprime une manière d'agir et d'être dans le monde qui doit être reconnu par toutes les communautés. L'enseignement de l'espagnol langue étrangère (ELE) doit être accompagné de la connaissance de ces variantes sociolinguistiques, sans se passer de la variante standard, pour chercher l'intégration de l'apprenant au contexte, sans le risque de refuser ou d'être refusé par le milieu où il doit se débrouiller. Cet article a deux objectifs très concrets: montrer quelques-unes des variables de l'espagnol en Colombie, les situant en tant qu'expression de groupes culturels du pays, qui méritent d'être reconnus avec leurs caractéristiques; et estimer les variantes comme objet de travail dans des contextes académiques, en revendiquant leur importance dans le contexte national. .

Mots clés: mode de vie, variante, système, cognition sociales, marché linguistique, symbolisation, réseaux sociaux, apprenant $d$ ELE.

\section{Variantes do espanhol colombiano e seu efeito no ensino do espanhol como língua estrangeira. Marco sociolinguístico}

\section{Resumo}

$\mathrm{Na}$ construção da língua como sistema interativo se requerem duas grandes entidades: um sistema formal e a sociedade, com todas suas cargas ideológicas e culturais, que lhe dão identidade ao sistema e o caracterizam em suas particularidades simbólicas. 
Este macrossistema social e linguístico apresenta variantes nos distintos contextos onde se coloca em cena e, por seu caráter idiossincrático, expressa um modo de atuar e estar no mundo que deve ser reconhecido por todas as comunidades. 0 ensino do Espanhol como Língua Estrangeira (ELE) deve estar acompanhado do conhecimento dessas variantes sociolinguísticas, sem prescindência da variante padrão, para buscar a integração do aprendiz a contexto, sem o risco de rejeitar ou ser rejeitado pelo meio onde lhe toque desenvolver-se. 0 presente artigo tem dois objetivos muito concretos: mostrar algumas das variantes que tem o espanhol na Colômbia localizando-as como expressão de grupos culturais do país que merecem ser reconhecidos com suas características e valorizar as variantes como objeto de trabalho dentro de contextos acadêmicos reivindicando sua importância no contexto nacional.

Palavras chave: modo de vida, variante, sistema, cognição social, mercado linguístico, simbolização, redes sociais, aprendiz de ELE. 


\section{Introducción}

La enseñanza del Español como Lengua Extranjera (ELE) ha estado transversalizada por la discusión acerca de la trascendencia 0 intrascendencia del sistema formal, la preponderancia o insignificancia de los aspectos sociales y culturales y la función que esos factores desempeñan en el proceso de enseñanza y aprendizaje de una lengua extranjera en el contexto académico. Es claro que el evento académico no es solo un proceder metodológico; debe tener un fundamento teórico que enmarque el proceder del docente de manera que los aprendientes de la lengua obtengan los mayores beneficios representados en la fluidez y eficacia comunicativa en las interacciones dentro del contexto en que participa. Para el caso específico del español como lengua extranjera en Colombia nos preocupa, en este trabajo, el tratamiento que se les daría a las distintas variantes que presenta el español en el país para ser acogidas y consideradas incisos trascendentales en la estructuración de un programa académico donde necesariamente han de integrarse el sistema formal de la lengua y los otros sistemas y gramáticas, como lo son lo social y lo cultural. Teniendo en cuenta lo anterior, el Instituto Caro y Cuervo, institución rectora de los estudios del español en Colombia, realizó esta investigación, que tenía como objetivos la caracterización de algunas variantes del español en Colombia y la importancia de ser reconocidas como parte integrante de la estructuración programática de los cursos de elE que se ofertan en Colombia.

\section{Metodología}

Para realizar este trabajo se requirió de una recolección de muestras tomadas de programas televisivos y de radio de algunas regiones del país, en las que se entrevistó a hablantes nativos de la región, interrogándolos acerca de su problemática regional y local. Se utilizaron, asimismo, muestras regionales tomadas de páginas de internet donde se consignan formas de habla; de ahí se tomaron las muestras correspondientes al sur del país, cuyo uso se contrastó posteriormente con el de hablantes adultos de la variedad en charlas informales.

\section{La lengua: sistema de subsistemas sociofuncionales}

La lengua, como todo sistema social, ha sido construida por el hombre en su devenir con el propósito de servirse de ella para cumplir eficazmente las distintas tareas que la 
realidad y sus necesidades le imponen. Podría pensarse que ha estado presente en todos los eventos en los que la humanidad ha participado para adecuar en primera instancia el entorno físico inmediato a las condiciones que su bienestar le pide. Esto podría inducir al error de considerar que, dado que todos los entornos físicos son diferentes, todas las lenguas tienen una estructura particular y única, en consonancia con las particularidades de cada contexto. Esa consideración es relativamente cierta y es el objeto de análisis de estas líneas.

No hay la menor duda de que las lenguas son variables en sus estructuras y en sus usos y de que esta estructuración y variabilidad están determinadas por unas condiciones específicas del medio, que construye en cada comunidad una cosmovisión internalizada en los hablantes-usuarios a partir del mismo momento de su nacimiento y configura su "modo de vida". Ese conjunto de conocimientos, que se adquieren poco a poco e inconscientemente, a la larga constituirán lo que se ha llamado la "cognición social", que, podría decirse, es la depositaria de todos los esquemas indispensables para desenvolverse adecuadamente en el contexto social y cultural, dentro del cual se participa en eventos y relaciones con otras personas. Se entiende que los comportamientos de todo orden no son simplemente actividades que se llevan a cabo motu proprio por los miembros de un conglomerado social; ellos obedecen a esa "cognición social", que, como se infiere, es un sistema internalizado que rige y norma esos comportamientos.

No se puede decir con certeza cuál es la naturaleza de esa "cognición”, pero se impone pensar que es una estructura de corte sicológico y social que subyace en la mente de los miembros de una misma comunidad a la manera de parcelas cognitivas compartidas, como lo aseguran Sperber \& Wilson (1986). De la misma manera, no puede dudarse de que existe en la mente de los hablantes un conocimiento compartido que, además de social, es también sicológico y lingüístico, común a todos los usuarios de una lengua, que requiere materializarse a través de mecanismos sicológicos, nerviosos, fisiológicos y experienciales para cumplir la función social para la que se creó e institucionalizó. Ese conocimiento compartido es el sistema lingiústico, que, a pesar de no tener una existencia independiente de las otras dimensiones humanas, de ser de naturaleza dinámica e interactiva con procesos sociales y culturales, sí tiene una lógica propia que se mantiene relativamente incólume a pesar de la amplia gama de variaciones del comportamiento lingǘstico, dentro de ciertos estándares que tiene el sistema. Esto muestra la necesidad de postular la lengua como la confluencia de principios estructurales y contextuales que se correlacionan para dar como resultado la configuración de un macrosistema integrado que no solo forma parte de la vida de las comunidades sino que es la vida misma. 
El anterior es uno de los principios fundamentales actuales de la lingüística; hoy esta no se puede concebir como un agregado de disciplinas sino como una inter-relación disciplinar en que se integran ciencias aparentemente tan disímiles como la sicología, la economía, la etnografía, la antropología, la sociología, etc., todas ellas con aportes valiosos para tratar de dar explicaciones sobre la lengua y la vida misma. Si se atiende a lo anterior, se tiene que aceptar a la sociolingǘstica como el epicentro de la ciencia de la linguística; es decir, toda lingüística es necesariamente sociolingüística. Es apenas obvio, entonces, que ninguno de los sistemas o de los subsistemas de que consta la lengua puede estudiarse independientemente, aparte de los otros, como lo hizo durante tantos años el estructuralismo con la gramática interna o sistema formal, pretendiendo dar razón de la lengua como un sistema ideal, desprovisto de las aparentes inconsistencias del habla o de la actuación lingüística; metodológicamente, sin embargo, este propósito es válido dada la complejidad del sistema interno y la importancia vital que tienen sus subsistemas como fundamentos de la lengua integral y la incidencia de cada subsistema en la descripción y el análisis de la misma en cuanto organismo que subyace a todas las prácticas sociocomunicativas.

Este organismo, objeto de la lingüística interna o microlingüística, está formado por los subsistemas gramaticales fonológico, morfológico, sintáctico y semántico, cuyo funcionamiento sincronizado permite la utilización coherente de los fonemas, las palabras, las oraciones y los significados en la comunicación interpersonal o en la expresión del pensamiento. Cada uno de estos subsistemas contribuye, con sus piezas y regulaciones, al funcionamiento del sistema como globalidad sincronizada, aportando sus unidades en forma acoplada, ya que cada uno tiene funciones específicas que cumplir dentro del sistema global. De la misma manera, como cada una de las partes del organismo humano tiene unas funciones que realizar y un lugar dentro de la estructura general del cuerpo, se podría decir que el sistema de la lengua tiene en las palabras uno de sus constituyentes fundamentales en el mecanismo lingüístico, con sus correspondientes formas, funciones y funcionamientos.

\subsection{El subsistema fonológico de la lengua}

Está formado por los fonemas, que son considerados abstracciones formales cuya presencia o ausencia incide en el significado de las expresiones, pero que en sí mismos carecen de significado. Su valor fonológico se capta cuando se oponen a otro fonema. Si, al hacer la oposición, la palabra cambia de significado, allí en ese cambio de significado se evalúa el valor fonológico de la unidad. Ejemplo: las palabras /máta/ y /áta/ se diferencian únicamente en un segmento, diferencia que hace que haya cambio en el significado, lo que lleva a inferir que en español existen dos fonemas $-/ \mathrm{m} / \mathrm{y} / \mathrm{l} /$ - cuyo intercambio modifica 
el significado. Otro tanto podría decirse de los otros fonemas del sistema fonológico del español o de cualquier lengua del mundo.

Este subsistema fonológico ha sido el más vulnerado histórica y sincrónicamente. Con motivo de las migraciones españolas del siglo XV, el español, como lengua, también migró y, al relacionarse con las lenguas nativas sur y centroamericanas, sufrió transformaciones y acomodaciones consecuencia de las interferencias lógicas entre los sistemas. Fue así como en Suramérica se neutralizó el fonema $/ \Theta /$, interdental fricativo sordo, reemplazado por el fonema /s/, alveolar fricativo sordo. Hoy en Colombia está en proceso de neutralización el fonema palatal, lateral, sonoro de llanta, callar, sellado, etc., característico de cundiboyacenses, huilenses y santandereanos, por el palatal fricativo sordo de yuca, apoyo, etc. Como se ve, la fonología no es un sistema autónomo: hay hechos sintópicos y diacrónicos, entre otros, que hacen que el sistema interno cambie como consecuencia de fuerzas extrasistémicas, lo que da lugar a variantes fonológicas y fonéticas íntimamente relacionadas; de ahí que algunos autores consideren la existencia no de un sistema fonológico y otro fonético mutuamente excluyentes sino un sistema fonéticofonológico cóncavo-convexo en sus configuraciones. Las variantes fonológicas, que afectan el sistema interno, son muy limitadas y se dan muy lentamente, con el correr de los siglos, lo que no ocurre con las innovaciones fonéticas, que son constantes y se dan a cada instante en la conversación dependiendo de muchos factores que se analizarán más adelante.

\subsection{El subsistema morfológico}

Tiene por objeto el estudio de las palabras, su estructura y su funcionamiento. La palabra morfología se deriva del griego morphé 'forma' y logía 'tratado' y se utilizó por primera vez en el siglo XIX para referirse a la forma y la estructura de los organismos vivos: animales y plantas. Posteriormente fue adoptada por la linguística para designar la estructura interna de las palabras utilizadas en cualquier lengua del mundo. Esta ciencia se originó en los estudios de Jan Baudouin de Courtenay, quien afirmaba que las palabras estaban formadas por raíces y afijos. En el campo de los estudios del lenguaje se ha de entender estrictamente como la rama de la lingüística que se ocupa de estudiar y analizar la organización interna de las palabras de todas las lenguas del mundo, los componentes de las mismas y sus posibilidades de transformación.

El papel de los estudios lingüísticos ha cambiado de acuerdo con las corrientes de las épocas; así, Leonard Bloomfield, lingüista norteamericano de mediados del siglo XX, la considera una ciencia esencial en los estudios del lenguaje, mientras que la Gramática Generativa Transformacional (GGT) de Noam Chomsky la considera un componente marginal 0 , a lo sumo, complementario dentro del sistema de la lengua. Una ciencia 
muy relacionada con la morfología es la sintaxis, que se ocupa de la disposición de las palabras para formar frases u oraciones. Entre ambas ciencias existe una relación íntima, hasta el punto de que existe una disciplina resultante de la correspondencia necesaria entre ellas: la morfosintaxis. Algunos autores llegan a afirmar que la morfología también puede entenderse como una sintaxis de la palabra y la sintaxis como una morfología de la oración, lo que evidencia el carácter sistémico integral de la lengua. Se podría decir que la morfología es la disciplina que se ocupa de la "gramática de la palabra" mientras que la sintaxis se puede definir como la "gramática de la oración".

La morfología, definida también como la ciencia que estudia las formas que adoptan las palabras en un contexto, se ocupa de:

a. identificar los elementos constitutivos de las palabras, es decir las partes en que pueden dividirse

b. clasificar dichos constituyentes

c. precisar la función que cumplen en la palabra

d. determinar cómo están distribuidos dentro de la palabra

e. formular las reglas que expliquen dicha distribución

f. explicar cómo se utilizan esos elementos en la formación de nuevas palabras.

La sintaxis hace lo mismo, pero dentro del campo de la oración.

La morfología, no obstante, no se ocupa de todas las palabras o de todas las categorías gramaticales; centra su estudio en las categorías gramaticales modificables contextualmente como el sustantivo, el adjetivo, el verbo, el artículo y los adverbios, derivados que varían según el contexto lingüístico y cultural. Dressler (1986) plantea la existencia de la morfopragmática como la parte de la morfología que se encarga de estudiar los efectos pragmáticos de la aplicación de las reglas morfológicas en un contexto de palabra. Es decir que la variación, como proceso lingüístico, también se da en el campo de la morfología; de ahí que algunas comunidades utilicen unos alomorfos en lugar de otros. En muchas regiones de Colombia se dice, por ejemplo: "Me voy porque me cogió la tarde", mientras que en algunas provincias de Santander del Sur se oye: "Me voy porque me cogió el tarde", hecho morfológico que podría explicarse solo a partir de unos factores culturales. Podrían darse otros ejemplos: en Chile es común oír la palabra gatito mientras en Colombia se oye gatico: se observa, pues, un cambio morfológico de $\{$-it- $\}$ por $\{$-ic- $\}$ sin ningún condicionamiento lingüístico específico para expresar esa equivalencia semántica; se trata de hábitos lingüísticos de una comunidad de habla o grupo isolectal. Volviendo al contexto colombiano, en las áreas rurales del Chocó se oyen expresiones como: "Yo no 
te vire" o "Yo no te vire, no", en que la doble negación y las paragoges que se observan obedecen posiblemente a factores heredados de una lengua de sustrato africano.

Es importante notar la correlación que se da entre lo morfológico y lo sintáctico, es decir que tanto las variantes morfológicas como las sintácticas obedecen a factores muchas veces contextuales que identifican una región, un estrato, un nivel educativo, etc., e inciden necesaria y suficientemente en la materialización de formas lingüísticas propias de ese contexto. No se puede desvincular de este hecho el subsistema semántico, preocupado -a muy grandes rasgos- por el significado de las palabras, su surgimiento y sus cambios. La semántica se convirtió con el tiempo en la "pariente pobre" de la lingüística, y hoy la preocupación por el significado ha pasado a ser objeto de otras ciencias, como la filosofía, la pragmática y la semiolingüística. La filosofía ha hecho grandes aportes, aunque el más grande fue hecho por Ludwig Wittgenstein con su máxima: "El significado de una palabra es su uso". A partir de ella, el significado ha dejado de un aspecto marginal del lenguaje para convertirse en la preocupación de la lingüística, de la pragmática, de la misma semántica con nuevos enfoques, lo que ha dado lugar a pensar en el "sentido contextual" como la transformación del significado cuando abandona el nicho del sistema e ingresa al contexto situacional para adoptar su dimensión social.

\subsection{Subsistema semántico-discursivo}

La semántica, entonces, ha tenido que traspasar el nivel de la palabra aislada, de lo lexical, de la oración, para profundizar hasta niveles más globales, macrolingüísticos, como el acto de habla, el discurso y la enunciación. La oración, por ejemplo, ha perdido su trascendencia para dar paso al enunciado y al discurso como las unidades básicas donde la lengua adquiere su dimensión social, expresiva, pragmática. En estos momentos, es imposible entender la lengua como un código en sentido estricto, como lo hicieron muchos lingüistas siguiendo la línea de Roman Jakobson; las ciencias lingüísticas preocupadas por el sentido han demostrado que los significados son unidades ideales, en cierta forma, metafísicas, que adquieren nuevas dimensiones en el contexto situacional de la conversación o del discurso. Un ejemplo simple, pero muy diciente, que ilustra lo anterior lo vemos en el siguiente caso.

Contexto microinteraccional: dos hermanas están en la casa y cada una de ellas tiene una tarea que cumplir en las labores domésticas por un acuerdo al que han llegado con la mamá. Y ocurre el siguiente diálogo:
A: ¡Pásame los platos!
B: ¡Estoy barriendo! 
Si $A$ le dice a $B$ que le pase los platos, es lógico que $B$ le responda algo relacionado con el tema de la conversación como: "Bueno", "Ya voy", "Espera un momentico", pero le dice que está barriendo, lo que aparentemente no tiene que ver con la orden dada 0 el pedido hecho; sin embargo, a partir de la situación real que se vive en el instante del pedido, $A$ hace una serie de movimientos inferenciales que le permiten inferir que $B$ no le dice realmente que está barriendo sino que se niega a pasarle los platos, dados unos presupuestos aceptados por ambas jóvenes. La respuesta que infiere $A$ es: "iNo!" Pero el código como tal no puede dar razón de estas implicaturas; hay que recurrir a instancias distintas a las microlingüísticas mismas para encontrar esa respuesta. Para responder, $B$ se apoya en el acuerdo a que han llegado y este es el garante que subyace y que hace que el diálogo se dé en esos términos.

Como el anterior, podrían darse otros ejemplos. Lo que se patentiza, como se ha dicho, es, primero, la relación integral que se da entre los distintos niveles formales de la lengua y el contexto, y, segundo, el papel fundamental que desempeñan el sistema, en la codificación y la decodificación de la oración, y el contexto, en su producción e interpretación cuando se transforma en enunciado. Esto hace que se planteen dos dicotomías fundamentales: oración-enunciado y decodificación-interpretación. La oración y su decodificación se encuentran en el plano estrictamente gramatical formal y el enunciado y la interpretación en el plano contextual, que también podría llamarse "gramática de la realidad objetiva y cultural".

En toda comunidad, un individuo común y corriente se involucra constantemente en distintas situaciones de habla, alternando sus papeles sociales como gerente, padre, profesor, vecino, obrero, etc. Cada uno de estos roles le determina una forma de hablar y de actuar, lo que le genera la necesidad de adaptarse a las situaciones mediante respuestas adecuadas, hecho que debe caracterizar a todos los miembros de una comunidad, lo que permite asegurar que existe lo que podría denominarse una "competencia social" que se adquiere de manera simultánea con la competencia linguística chomskiana.

Esta competencia social, no susceptible de ser explicitada mediante reglas -como sí se ha hecho con la gramática lingüística-, se manifiesta de diferentes maneras en diferentes situaciones comunicativas y puede considerarse un repertorio de actuaciones que debe tenerse en cuenta para confirmar la adscripción del individuo a su entorno social pero, sobre todo, para producir en el interlocutor efectos que, en primera instancia, favorecen la propia autoestima, confirman la imagen social positiva que todo individuo tiene de sí mismo y promueven nuevos encuentros sociales satisfactorios. Esta, que podría denominarse "competencia social interactiva", se entiende como "saber" establecer y mantener relaciones interpersonales mediante el uso de los repertorios lingüístico y 
paralingüístico, gestos, por ejemplo, necesarios para afrontar situaciones comunicativas efectivas y exitosas, de ocurrencia común en el ámbito cultural. Siendo este "saber" una construcción simbólica propia de cada entorno, no es difícil colegir que está configurado por un sistema de reglas que estructuran una "gramática social" tan importante y válida como la misma gramática formal de la lengua.

En las prácticas sociolingüísticas, dichas gramáticas -la formal de la lengua y la social interactiva- se complementan, se necesitan, evidenciando la correlación de lo mental, lo cognitivo y lo social en los estudios pragmáticos y semántico-discursivos. En otras palabras, es lógico pensar que la lengua, como sistema, se ha construido en los procesos interactivos de una comunidad con un propósito intersubjetivo; es decir, la inmanencia del sistema linguístico no es exclusivamente abstracta sino además social, como tanto se ha recalcado. La gramática de lengua, entonces, no es producto del querer de los gramáticos, sino un sistema deducido de las actuaciones de los individuos en sus usos cotidianos. Por contera se descarta que los estudios pragmáticos sean meras prácticas sin fundamentos mentales y cognitivos; por el contrario: tienen su razón de ser, en primera instancia, en la racionalidad, la capacidad linguística y la necesidad.

De acuerdo con lo anterior, toda sociedad debe ser considerada un constructo de sistemas de reglas abstractas e interaccionales, linguísticas, sociales y culturales, inclusive políticas, que se reconocen en todas las secuencias de intercambios comunicativos. Esto demuestra la íntima relación entre el uso de la lengua, la sociedad y la cultura, procesos que se interretroalimentan y se requieren en la constitución y práctica del ser social. En términos gráficos, se podría asegurar que el uso de la lengua es un evento social y cultural y todo evento cultural tiene que darse a través del uso de la lengua. Es en este marco de lengua-sociedad y cultura donde se patentizan las variaciones sociolingüísticas, más 0 menos ilimitadas, que puede tener un sistema lingüístico, donde la gramática de la lengua o gramática interna conserva su invariabilidad, pero los otros factores materializantes sociedad y cultura- median en su transformación, concurriendo en la materialización de enunciados o actos de habla y del discurso para evidenciar su posición en el mundo y las condiciones en que el mismo ha sido cognitivizado. Este es uno de los hechos más complicados para los aprendientes de una lengua extranjera, dado que se debe no solo ingresar en el sistema interno de la lengua que se aprende, sino también enfrentar muchos otros aspectos relevantes y tener en cuenta una cantidad de variables necesarias que de alguna manera pueden afectar el aprendizaje o la adquisición de la lengua, según el caso. 


\section{Subsistema social-lingüístico}

Retomando la variación, puede decirse que es un hecho universal, es decir que todas las lenguas del mundo sufren modificaciones enunciativas determinadas por factores sociales, económicos y culturales, que son los que sustentan y dinamizan la lengua en sus particularidades discursivas. Estas transformaciones son idiosincráticas y están asociadas con las condiciones objetivas propias de un grupo social que se diferencia de los otros por la forma institucionalizada como se vive y se hace uso de la lengua -forma adoptada como constituyente simbólico de su identidad-. Sin embargo, esto no debe considerarse exclusivamente un hecho global; todas las personas usan variantes o alternativas sociolingüísticas de una misma lengua que dependen del contexto inmediato en que se encuentren inscritas en un momento determinado, lo que puede interpretarse como que todas las variantes de la lengua están global y microcontextualmente determinadas o que, como decía el maestro Luis Ángel Baena: "La lengua no se da en el vacío". Es importante anotar que el aprendizaje o la adquisición de una segunda lengua o una lengua extranjera le exige al aprendiente familiarizarse con las distintas formas de hablar esa misma lengua y ser capaz de desenvolverse en los distintos contextos donde le corresponda actuar como actor de comunicación. Esto, en últimas, implica que la enseñanza de lenguas extranjeras debe contemplar dentro de sus contenidos un proceso de conocimiento, adaptación cultural y familiarización del nuevo usuario con las gramáticas culturales particulares a fin de que sus participaciones linguísticas sean adecuadas a las situaciones que viva en una comunidad de habla o grupo isolectal.

Consideramos oportuno traer a colación el concepto de comunidad de habla que trae Francisco Moreno Fernández (2009, p. 23): "Una comunidad de habla está formada por un conjunto de hablantes que comparten al menos una variedad linguística, unas reglas de uso, una interpretación de ese uso, unas actitudes y una misma valoración de las formas linguísticas". Los miembros de una comunidad de habla se reconocen en sus usos, en sus prácticas simbólicas y en las valoraciones que hacen de esos usos, lo que los identifica creando vínculos fuertes entre ellos y resistencia a presiones externas que puedan provocar innovaciones culturales que afecten las normas vernáculas y la solidaridad grupal.

Estas comunidades de habla corresponden a diferentes zonas geográficas, cuya importancia o categoría en el contexto nacional está en íntima relación con el poder político y la riqueza. No es de extrañar que la valoración que se hace de la forma de hablar de la gente se equipare con la región de su proveniencia. En Colombia, la capital de la República, Antioquia, el Eje Cafetero, el Valle del Cauca y la Costa Atlántica se caracterizan por su riqueza, su importancia en el mercado internacional, la variedad de sus recursos y su participación en la burocracia a escala nacional, mientras que hay otras regiones, como 
la del Pacífico y la del sur, que se han estigmatizado con estereotipos poco favorables por razones históricas, pero sobre todo por no aportar lo suficiente y no tener la injerencia que tienen las otras. Puede parecer extraño, pero la verdad es que esa trascendencia política y económica determina la evaluación que se hace de la variedad de español que se utiliza en las regiones. En Colombia, las variantes lingüísticas de mayor prestigio manifiesto son la bogotana, la "paisa" y costeña. A manera de anécdota, en Colombia se habla de "paisañol" y "costeñol" para referirse a las variantes del español que se utilizan en las dos últimas comunidades de habla. Mientras las formas de habla de las primeras regiones gozan de un prestigio manifiesto, las segundas tienen un prestigio encubierto, y se podría decir que sus variantes están confinadas a ser utilizadas sintópicamente.

Lo anterior es un hecho imposible de ignorar en la enseñanza de ELE. Se ha dicho que Colombia es una nación de regiones, cada una con sus propias características y sus propias manifestaciones culturales. Aunque no se deben ignorar las variantes estándares de mayor prestigio en el país, los aprendientes de ELE deben conocer la existencia de otras manifestaciones, mal llamadas "subestándares", posiblemente estigmatizadas, que merecen conocerse y estudiarse no como expresiones de una Colombia marginal sino como variantes sociolingüísticas representativas de comunidades con idiosincrasias particulares que también tienen derecho a existir y a ser respetadas.

De lo dicho se puede deducir fácilmente que las variantes lingüísticas de una lengua son necesariamente sociolingüísticas y no están en distribución libre, o sea que no son reemplazables unas por otras en el mismo contexto de habla sino que están en distribución complementaria, es decir que las variantes se desplazan unas a otras, determinadas por condiciones sociales y culturales propias del contexto interaccional, tales como la distancia social que media entre los interlocutores, el contexto físico, el asunto del que se trata y otras variables que se tratarán en detalle más adelante. Lo anterior se podría ilustrar con un ejemplo muy claro. Véanse las siguientes expresiones:

1. El señor estaba enojado.

2. El señor estaba verraco.

3. El señor se arrechó.

4. El señor se salió de la ropa.

5. El señor se puso digno.

6. Al man se le subieron los apellidos

7. El mancito se embejucó

8. El cucho se despelucó.

9. El mandril se salió de los chiros

10. El man se enfurruscó. 
11. Al hombrecito se le subió el tigre.

12. El mancito se delicó.

Es posible que, desde el punto de vista referencial, las expresiones anteriores se consideren "sinónimas" o posiblemente "semánticamente equivalentes", pues simplemente ha habido una "substitución" lexical, como podría decir la lexicología, dando explicaciones de corte geográfico. Sin embargo, desde el punto de vista sociolingǘstico no es posible asegurar esto, dado que a cada una de esas expresiones subyacen informaciones de tipo social, demográfico, cultural y etario que es imposible ignorar en caso de querer penetrar en su sentido, lo que es casi inmediato en la comunicación, en el momento mismo de la interacción. Todo esto induce a pensar que no debería hablarse de variantes lingüísticas sino de variantes sociolingüísticas en sentido estricto. A partir de los usos de las formas anteriores se podría hacer una radiografía explicativa de lo que es Colombia, sus regiones, sus modos de vida, sus idiosincrasias, sus múltiples culturas y estilos de habla, para lo cual se tendrían que dar explicaciones a quienes aprenden el español como lengua extranjera, sin lo cual se quedarían únicamente con una forma estándar y prestigiosa, algo cuya consecuencia lógica sería caer en fosilizaciones provocadas por el sistema de enseñanza. Desde el punto de vista didáctico, y con el fin de evitar falencias de este tipo, podría pensarse en llevar a las clases formales a hablantes nativos de esas variantes del español o, en su defecto, recurrir a programas de los medios de comunicación social, corriendo el riesgo de no lograr el objetivo dado que la mayoría utilizan formas prestigiosas o estandarizadas por razones que ellos mismos manejan.

La dialectología, sobre todo en las décadas anteriores a 1960, se había preocupado por la variación; sin embargo, solo se llegaba a hacer una ubicación geográfica o regional, sobre todo en zonas rurales, de las variantes para después visualizarlas en mapas de isoglosas en que se mostraban los dialectos y sus fronteras dentro de una región o un país -objetivo que requería faraónicas inversiones de tiempo y dinero-. La construcción de dichos mapas era la meta de esos estudios dialectológicos; hasta ahí llegaba su estudio. El estructuralismo, por su parte, llegó un poco más allá al tratar de describir los fenómenos fonéticos, morfológicos, sintácticos y -muy poco- los semánticos desde el mismo sistema formal, sin profundizar en los aspectos extralingüísticos o contextuales, que es adonde pretende penetrar la sociolingüística con la participación de ciencias como la antropología y la sociología, principalmente, con las que construye su objeto de estudio y su marco conceptual. De todas maneras, no pueden ignorarse las dos tendencias mencionadas que, de muchas maneras, dieron la oportunidad de reconocer la "diversidad dialectal" en muchos países, donde cada variante tiene sus formas y razones específicas de uso, prestigioso 0 no. 
Para analizar las distintas formas del español en Colombia, es pertinente mencionar a dos lingüistas colombianos de grata recordación: los profesores-investigadores José Joaquín Montes Giraldo y Luis Eduardo Álvarez Henao, quienes, uno en Bogotá y el otro en el Eje Cafetero, se preocuparon por estas variantes desde diferentes posiciones teóricas. El valioso trabajo de ambos, en cierta forma, alienta este intento de acercamiento a la lengua.

En primera instancia, interesa analizar algunas variantes utilizadas en el habla corriente en Colombia, y para comenzar es viable mirar ciertos fenómenos de dicción fonéticos- muy frecuentes en el habla. La linguística y, más concretamente, la fonética han ubicado ciertas transformaciones, a las que ha denominado "metaplasmos", que se definen como transformaciones del significante sin cambio del significado referencial. Se insiste en el hecho de que se habla de un significado referencial y no de las implicaciones de su uso. El tratamiento de la fonética es estrictamente intralingüístico, no sociolingüístico. Ejemplos de estos metaplasmos son, entre otros, aplanchar, déntrese, aguao, cunclillas, agüela, estógamo, estuatua, incontrar, fuistes, infriar, enjermo, diabetis, medecina, proesor, restrinción, homosesual, herver, comelón, tualla, cónyugue, almóndiga, güeno, jinca, cajé, y hay muchísimos otros que, en apariencia, son usados por personas socialmente marginadas, pero la verdad es que algunas de estas variantes se les oyen a personas de condiciones educativas y sociales que harían presumir que usan formas de prestigio abierto. La fonética misma, y tal vez la dialectología, ha clasificado estos metaplasmos desde adentro del sistema, describiendo fenómenos como asimilaciones, disimilaciones, prótesis, epéntesis y metátesis, entre otros, sin alcanzar un nivel explicativo.

Es importante anotar que, muchas veces, los usuarios pertenecientes a algunos sectores sociales o nativos de algunas regiones del país, a pesar de tener formación profesional, no optan por la variante prestigiosa sino que continúan usando la variante regional, como es el caso de los comunicadores sociales de las costas colombianas, quienes utilizan, entre otras, la variante aspirada del fonema alveolar fricativo sordo /s/ al final de sílaba trabada o de palabra o la eliden, como ocurre en casos como [ehtói], [kapáh], [kapá], [míhmo], o de la elisión de la alveolar vibrante múltiple sonora en posición de final de palabra, como en el infinitivo de los verbos [kantá], [bailá]. Estos hechos fonéticos se muestran en una entrevista a un habitante del municipio de Puerto Meluk (Chocó), hablante nativo de la variedad pacífica, de aproximadamente 30 años. Esta entrevista fue realizada el martes 5 de junio de 2012 por dos periodistas del canal regional Telepacífico en la región del Baudó, en jurisdicción del municipio mencionado y se transcribe a continuación:

Locutor 1: Los habitanteh de Puerto Meluk, piden que máh empresah de tranhporte prehten el servicio de tranhporte dehde y hacia el municipio. 
Locutor 2: Siendo la vía Ihmina - Puerto Meluk una alternativa para salir al mar Pacífico por Pizarro, eh precario el sihtema de tranhporte terrehtre entre estah doh poblacioneh, pese a que la carretera ehtá en buenah condicioneh. Loh viajeroh ehpresan su preocupación por la falta de vehículoh en la ruta, lo que se convierte prácticamente en una odisea para hacer el recorrido dehde y hacia Puerto Meluk.

Entrevistado: Eh un poquito incomprensible entendiendo que el flujo de pasajeroh eh demasiado abundante por ehta zona. Hay una sola empresa trahportando pasajeroh y no tiene un horario suficiente pa que la gente pueda trahportarse debida y adecuadamente; eh decir, llega uno aquí a Puerto Melú y muchah veceh tiene que ehperá treh o máh horah pa podé viajá. Generalmente sucede lo mihmo de venida dehede Ihmina pacá, o sea creemoh que lo máh importante sería, que sa o lah empresah, pongan una ruta de menó tiempo para que así loh pasajeroh puedan andá máh frecuentemente.

Locutor 1: ¿Qué pasa con la gente que viene de lah comunidades de arriba y abajo y llegan aquí y no encuentran tranhporte? ¿Qué pasa con elloh?

Entrevistado: Pueh muchoh de elloh tienen a veceh problemah inclusive hahta de alojamiento cuando leh toca quedase aquí, juhtamente esperando trahporte sea para salí o pa entrá inclusive.

Locutor 2: Y cuando viene alguna persona de otra comunidad, ¿cómo hacen?

Entrevistado: Bueno, eso sí ehtá solucionado porque aquí, aunque lah ambulanciah no permanecen conhtantemente acá, al menoh hay un servicio de ambulancia bahtante permanente.

LocuTor 1: Diariamente ¿cuántoh buseh ehtán entrando acá?

EnTREvistado: Acá ehtán entrando doh o treh buseh, la empresa Ehpresosh del Pacífico ehtá enviando doh o treh buseh según la temporada.

Locutor 2: Vemoh mucho personal para viajar, mucha carga, ¿no?

Entrevistado: Eso sí eh una cosa muy frecuente, normalmente viajamoh con doh o treh maletah y eso hace que el equipaje se vuelva bahtante abundante.

LocuTor 2: Hahta el momento una sola empresa de buseh realiza loh viajes variah veceh durante la semana, pero por tratase de una vía tan transitada, pa salí o llegá a Pizarro, o al municipio del Alto Baudó, la gran mayoría de personah no encuentran el trahporte requerío.

Aunque hay una diferencia muy grande entre el habla de los entrevistadores y la del entrevistado, se nota que los primeros, por pertenecer culturalmente a la región del 
Pacífico, conservan algunos rasgos fonéticos característicos de esa variedad. Como se decía, el nivel educativo alcanzado no ha borrado del todo el habla del Pacífico, conservándose la aspiración de la alveolar fricativa sorda /s/ -posiblemente el rasgo menos estigmatizado, precisamente por darse también en la Costa Atlántica, que tiene prestigio en el contexto colombiano por su importancia económica-. El entrevistado, por su parte, conserva intactas las variantes fonéticas y prosódicas de su entorno nativo, pues no participa directamente en el mercado lingüístico de la formalidad económica, como sí lo hacen los entrevistadores.

La literatura, sobre todo la novela, el cuento y la poesía costumbrista, se ha preocupado por las variantes en una perspectiva expresiva y poética; ejemplo de ello es un autor como Tomás Carrasquilla, con hermosos cuentos como "Dimitas Arias", entre otros, donde retrata naturalísticamente escenas campesinas antioqueñas y recurre a la lengua con este propósito. De la misma manera, Miguel Ángel Caicedo retrata en sus poemas costumbristas el habla del campesino chocoano en su contexto de selva y orilla. Tanto Carrasquilla como Caicedo muestran mil y una variantes fonéticas que ayudan a comprender el alma de Colombia en la dimensión folclórica más auténtica de sus regiones.

Pero no todo es pasado; recientemente, El Tiempo publicó en sus páginas el cuento "El mandao", de Nicolás Marrugo Silva, que se transcribe en forma literal:

- ¡Apúrese que está serenando! -gritaba el Coronel desde la terraza de la casona¡Límpiese y escúrrase ahí! Está embadurnao e barro. Lávese esos pies tan percudíos. Vea que su abuela ya trapió. Parece un pordiosero.

-¡Abuelo, no me regañe! Estaba comprándole doscientos pesos e aceite a buela Mina -respondió Gabito, agitado.

-¿Por qué no llevó paraguas? -insistió el exmilitar.

-Cuando salí el sol estaba bien picante -murmuró.

- ¡Tranquilina! Ojalá no le vaya a dá gripa a este pelao -replicó el Coronel.

-¡Apúrese pá fritá rápido estas tajás e plátano que me tengo que î́! -chilló desde la cocina la abuela.

- ¡Cálmate, Mina, dejá reposá a Gabito! Por andá corriendo se le reventaron las abarcas.

-¡Da gracias que no se escalabró! -enfatizó el abuelo.

-¡Fíjate tú! Te lo tengo advertío: ¡no corras cuando hagas el mandao! Pero ese bendito pelao no hace caso. ¿Dónde está el aceite? -preguntó la abuela. 
-¡Se me rompió la bolsita! - contestó Gabito.

-¡Mira tú esa vaina! - amalayó abuela Mina - ¡Cuál de los dos más despistao! ¡Tiene tu nieto a quien salí! -exclamó Tranquilina mirando fijamente al Coronel.

-¡Voy pa misa! Ustedes verán cómo se bandean.

No se pretende hacer un análisis literario: simplemente queremos resaltar algunos fenómenos fonéticos y morfológicos en el contexto macro y microlingüístico. En primera instancia se trata de una supuesta conversación sostenida entre los abuelos de Gabriel García Márquez, en la que se identifican los tres personajes por "utilizar" el costeñol o "superdialecto costeño", como lo denomina el profesor Carlos Patiño Rosselli (2000). La forma de hablar de los participantes dice que están en un contexto corriente donde se desarrolla el acto de habla regañar, que los actores del acto de comunicación son personas que no tienen un alto nivel educativo, que se mueven dentro de unas redes sociales de baja densidad y que no son de un estrato social alto. Todos ellos utilizan el costeñol, como se dijo, que es - como lo caracteriza Patiño Rosselli aludiendo a Montes- "un superdialecto costeño donde se registran particularidades que definen el español meridional (aspiración o caída de /-s/ posvocálica, neutralización o intercambio de /r/ y // implosivas, pérdida de /-r/ final y pronunciación velarizada de /n/" (p. 63). A esta información se le puede agregar el hecho de la elisión de la dental oclusiva sonora -/d/- en posición intervocálica, como se ve en [peláo], [adbertío] y otras; así mismo es evidente el apócope de la preposición para que se vuelve [pá], fenómeno presente no solo en el costeñol sino en la gran mayoría de las variantes regionales de Colombia. Llama la atención la prótesis del morfema gramatical verbalizador, prefijo $\{a-\}$, que utiliza el autor tratando de reproducir el habla regional al formar el verbo amalayar, muy extraño en variantes de otras regiones del país.

En el Valle del Cauca, sobre todo en Cali, Palmira, Candelaria y zonas circundantes, se presenta el fenómeno de la neutralización de /n/ en posición de final de palabra, que se materializa en bilabial nasal sonora [-m], como en pan, almidón, caimán, que se fonetizan [pám], [almidóm], [kaimám]. A propósito de las variantes de /d/, es interesante hacer una observación de sus variaciones fonéticas (alófonos). Además de fricación en posición intervocálica, hecho muy generalizado en Colombia, también se presenta su elisión, en las variantes de las costas, en la misma posición intervocálica. Pero en la variante del Pacífico, en el sector rural, con sus características de marginación, el mencionado fonema se neutraliza o intercambia con la vibrante simple /r/ o con la lateral sonora $/ /$, por lo que es común escuchar expresiones como: "Los daros ehtán calgaros", que alterna con: "Los daros ehtán calgaos". Por otra parte, en la variante pastusa - ¿"pastuñol"?-, el mismo fonema no sufre modificación alofónica en la posición intervocálica, hecho por demás 
bastante extraño dentro de las variantes del español en Colombia, incluyendo la variante utilizada en Bogotá, que se cataloga como la variante con prestigio manifiesto o abierto, en tanto que las variantes pastusa y pacífica, sobre todo, son las más estigmatizadas y de prestigio encubierto, más que todo por razones históricas y económicas, como se dijo. No se considera necesario seguir trayendo casos de variantes fonéticas de las distintas regiones de Colombia; lo que se ha pretendido es mostrar la correlación entre las variantes utilizadas por los usuarios de una lengua, su contexto y la identidad social, que desarrolla toda una serie de estrategias culturales y lingüísticas que permite la identificación de la membresía y la supervivencia del grupo.

Las variaciones morfológicas y sintácticas, como es lógico, también están determinadas lingüística y contextualmente. Las variantes morfológicas están relacionadas con cambios de morfemas gramaticales de género, número, pronombres y tiempo y con la conjugación de los verbos. Sin embargo, hay que hacer notar que las variaciones de naturaleza morfológica y sintáctica son menos frecuentes que las fonéticas, sin desconocer su existencia y papel identificatorio dentro de las culturas. Entre las variaciones morfológicas de mayor ocurrencia está la que se da con el pronombre de segunda persona del singular: usted, vusté, tú, vos, su persona, sumercé, muy utilizadas en Colombia. El pronombre vusté fue, en su momento, muy común como fórmula de tratamiento en las zonas "montañeras" de Antioquia; hoy, como consecuencia de los procesos de urbanización de estas comunidades, la forma ha caído en desuso y se considera un arcaísmo. Las otras formas son de uso frecuente, y se podría hablar de una territorialidad de cada una de ellas. El tú es muy utilizado en la zona Caribe, donde posiblemente sea la forma más habitual; otro tanto ocurre con sumercé, de común ocurrencia en la altiplanicie cundiboyacense, si bien también es común utilizar usted. A veces da la impresión de que el uso de estas formas está en relación con la distancia social que media entre los interlocutores. Su persona, por su parte, se considera una forma que caricaturiza la forma de hablar de los campesinos, sobre todo en los medios masivos de comunicación, pero es de uso muy esporádico en el habla corriente de Boyacá. Por su parte, el usted y el vos, en zonas como el Eje Cafetero, está en relación directa con la distancia social entre los participantes en conversaciones; entre mayor sea esta variable, o si quiere "marcar territorio", se utiliza el usted y, en caso contrario, el vos.

Otra variación morfológica está relacionada con la flexión de la primera persona del plural. Son frecuentes, en algunos sectores marginados, de bajo nivel educativo, expresiones como "cantábanos", "subíanos", "estábanos" por cantábamos, subíamos, estábamos. Es curioso, por decir lo menos, que se utilicen formas del pretérito imperfecto para expresar acciones del presente, como ocurre en: "Yo venía a pedirle el favor de que 
me prestara dos mil pesos" o: “Usted qué quería?", cuando la verdad es que son eventos que están sucediendo en el presente y debería decirse: "Yo vengo a pedirle el favor de que me preste dos mil pesos” y: “¿Usted qué quiere?” La variante se explica por factores contextuales, pues, para mitigarle el costo al destinatario, el emisor modaliza con formas indirectas e ilocutivas corteses.

En el sur de Colombia se oyen verdaderas joyas morfológicas, dignas de investigación. Así, en la región pastusa se oyen expresiones como: "Darásmelo cuidando" 0: "Dárasme cuidando estico", que significa que tú me estás haciendo un favor que yo recibo si tú me cuidas este elemento. "Me estaba aguantando semejante heladez", en donde beladez se refiere al clima demasiado frío característico de la región; en áreas campesinas de Santander la llaman "yelaje". "Ese señor es muy alborotista" se refiere a un señor que forma demasiado alboroto, desorden. "YYa llegaría la recomienda?", por encomienda, que es la palabra que se utiliza en el habla estándar. "Deme acomodando estico" es un pedido que hace el emisor para que le cuiden algo, y el uso del morfema \{-ic-\}, cumple una función modalizadora, atenuadora, dando a entender que lo que se va a cuidar es algo muy pequeño, que no ocupa mucho espacio y que no habrá que hacer mucho esfuerzo para cuidar lo dejado y que será por corto tiempo. "Cuidarasmelita" es un pedido que le hace el emisor a su destinatario para que le cuide algo; aquí vuelve a aparecer el diminutivo con la función de atenuador, dando a entender que al receptor no le costará mucho esfuerzo hacerle el favor solicitado. Morfopragmáticamente se podría analizar en los siguientes términos: el morfema lexical \{kuid-\}, del verbo cuidar, indica la acción por realizar; el morfema vocal temática del verbo de primera conjugación $\{-\mathrm{a}-\}$; el morfema de modo indicativo, tiempo futuro, aspecto imperfectivo $\{$-rá- $\}$, indica que la acción va a ser realizada; la flexión $\{-s-\}$ indica que es la segunda persona del singular la que va a realizar la acción; $\{$-me- $\}$ pronombre átono, objeto indirecto, indica que el emisor va a ser el beneficiado; el $\{$-it- $\}$ modalizador se tiene que interpretar como que el destinatario no tiene que hacer mucho esfuerzo para realizar la acción, y $\{-a\}$ indica que lo cuidado es un objeto cuyo género gramatical es el femenino. Al parafrasearse la expresión, podría afirmarse que el emisor dijo: "Compadre, por favor cuídeme esta maletica por un ratico mientras yo vuelvo; no me demoro". Es interesante notar el uso reiterativo del diminutivo con una función pragmática muy clara para el interlocutor, así como la de cada uno de los elementos morfosintácticos utilizados con el propósito de satisfacer los requerimientos para que se lleve a cabo el acto de habla con todas las condiciones de felicidad requeridas.

Las anteriores son expresiones que quien las escucha por primera vez no interpreta rápidamente debido a un uso morfosintáctico o morfopragmático bastante particular, debido posiblemente a un calco de formas equivalentes en su lengua de sustrato. También 
llaman la atención, en esta variante pastusa, la forma de despedida "cuidaraste" y la advertencia o recomendación que le hace la madre a su hijo con la expresión "manejarase bien". De paso, en los Santanderes se utilizan expresiones como: "Ese pingo es mucho lo toche" y: "Bucaramanga es una ciudad mucho lo bonita", de sintaxis un tanto distorsionada pero interpretable, que es lo fundamental en los enfoques comunicativos.

Las anteriores muestras, tomadas al azar y de forma asistemática, algunas de la página "Decirlo con acento pastuso", son expresiones que responden a unas variables sociales de que se ha hecho mención rápida pero que es interesante analizar para esclarecer su función en los usos comunicativos. Es un axioma de los estudios sociolingüísticos que toda expresión de la lengua que se usa se da en situación y contexto reales y que varía en función de factores específicos propios de cada comunidad. Entre estos factores se pueden ubicar el género, la edad, la clase social, el mercado lingüístico, las redes sociales, el nivel de instrucción, la profesión, la procedencia y el modo de vida -todos íntimamente integrados y correlacionados de la misma forma como se integra la vida personal de un autor, su modo de vida, en su obra literaria-.

El modo de vida es, sin duda, la variable más importante de los grupos ya que explica cómo están organizados, la configuración de las redes sociales y de solidaridad internas, y cómo se relacionan con las de otros grupos. Se supone que dentro de los grupos hay diferentes modos de vida relacionados con la forma como subsisten y con las prácticas culturales que sustentan su modo de producción y las relaciones familiares. Es de notar que, cuando son fuertes y consistentes, las redes sociales utilizan formas lingüísticas propias y características, mientras que, si son débiles y el grupo tiene poca fortaleza antropológica, emplean formas linguísticas de prestigio abierto, se acogen a normas linguísticas impuestas por el grupo de mayor influencia social, política o económica. El acogimiento de las variantes de prestigio abierto por estas comunidades obedece fundamentalmente a la creencia de sus miembros de que la utilización de las formas del prestigio abierto o de códigos elaborados facilita el ascenso social individual y de la comunidad en general.

Las características de las redes sociales son una consecuencia del modo de vida y se entienden como el conjunto de relaciones que se establecen entre los miembros de una comunidad con el fin de intercambiar bienes y servicios, establecer roles y mantener un modo de vida acogido por todos. Estas redes sociales tienen distintas dimensiones, dadas por su densidad y su complejidad, determinada la primera por el número de vínculos que tiene y mantiene cada individuo de la red; y la segunda, por el tipo de relaciones que existen entre los mismos; por ejemplo, si las personas tienen vínculos de consanguinidad, la red puede ser más compleja mientras que, en una relación comercial, la red puede ser más densa. Las redes complejas son generalmente más estables y conservan formas 
lingüísticas vernáculas que, a veces, corresponden a códigos restringidos mientras que las redes densas utilizan formas estandarizadas propias del mercado linguístico y su duración es fugaz.

El género como variable social es otro factor altamente incidente en el uso de la lengua; a pesar de los fenómenos educativos, sociales y políticos de los últimos años, y de su participación activa en el acontecer diario de la sociedad en su conjunto, la mujer ha conservado formas distintas de hablar que no connotan exactamente sometimiento al hombre, como se pensaba hace algún tiempo, sino que ellas se consideran una especie de guardianas de la institucionalidad cultural y, para efectivamente serlo, utilizan generalmente formas estandarizadas y de prestigio abierto a través de las cuales "se dan su lugar" y exigen reivindicaciones para no ser vulneradas y ser reconocidas en el rol que socialmente se le ha asignado. En una charla con una mujer profesional, uno de los autores de estas líneas la interrogó acerca del problema y ella respondió con mucha seriedad: "No me gusta hablar mal ni utilizar vulgaridades porque considero que estoy irrespetando el español". El conservadurismo lingüístico femenino está explicado en esta sabia respuesta, nacida de las autorregulaciones femeninas, acogidas por respeto a sí mismas. Por último, es de anotarse que las preferencias linguísticas de las mujeres no tienen una razón de ser en el sexo como categoría biológica sino en los patrones de crianza que se han utilizado y siguen utilizándose en los diferentes contextos sociales y culturales.

El mercado lingüístico es, sin duda, una de las variables más importantes para la catalogación prestigiosa o no de las hablas. Todas las personas de las comunidades se relacionan directamente o no con los medios de producción y para ello deben adoptar ciertos comportamientos que de alguna manera favorezcan su imagen positiva; uno de ellos es utilizar la forma normativa de la lengua que concuerde con, o se acomode a, la forma prestigiosa de hablar de los propietarios de esos medios de producción. Es claro que las exigencias de ese mercado lingüístico están en relación directa con la importancia del desempeño profesional o no del aspirante, ya que, de todas maneras, quien se quiere integrar al mismo debe dar pruebas lingüísticas de su calidad humana y para lograrlo hace las acomodaciones discursivas necesarias a fin de impresionar a quien lo va a vincular a la actividad laboral. Gregory R. Guy (1992, p. 57) define el mercado lingüístico como "el índice que mide específicamente cómo la actividad económica de los individuos, entendida en su sentido amplio, requiere, o está necesariamente asociada con su competencia en la variante socialmente legitimada o estándar". Este concepto hace pensar en cuestionamientos extralingüísticos como estos: ¿quién establece e institucionaliza la variedad estándar 0 prestigiosa?, ¿es un hecho lingüístico?, ¿es una imposición política, económica, social? 
La edad, por su parte, es otra variable incidente en el uso de la lengua y los sistemas paralinguísticos; los jóvenes usan la lengua de una forma muy particular, ya que generalmente son quienes introducen innovaciones lingüísticas y utilizan expresiones paralinguísticas amplias y enérgicas que, en la mayoría de los casos, son rechazadas por las personas de mayor edad, que consideran que las variantes de los grupos más jóvenes afectan negativamente la lengua, hasta el punto de hablarse de "corrupción" de la lengua y pérdida de valores ancestrales, hecho que estigmatiza su forma de hablar y la ubica socialmente como subestándar. No hay duda de que la forma de hablar de los jóvenes tiene determinadas marcas, particularmente en el léxico utilizado y en la gestualidad, como saludarse con fórmulas consideradas poco vernáculas, chocar los puños y otros juegos quinésicos. En Colombia, por ejemplo, la utilización de la palabra parcero, o de su forma apocopada parce, fue utilizada inicialmente por jóvenes de extracción popular y de estratos bajos; sin embargo, poco a poco ha penetrado otras esferas etarias y sociales y ya está escalando posiciones en estratos más altos y de personas con algún nivel de preparación académica, hecho que parece ser la tendencia general. La lengua, de todas maneras, se acomoda a las nuevas tendencias sociales, so pena de hacerse obsoleta y perecer. Otro tanto podría decirse de algunas formas seculares de saludo como "buenos días”, "¡cómo le va?”, “¿qué tal?”, etc., que han sido substituidas por fórmulas como “‘todo bien?”, o de fórmulas de despedida como "cuídese", que podría dar lugar a interpretaciones alejadas de lo usual.

Todo parece indicar que ese afán de innovación iconoclasta lingüística se da en una etapa de la vida, y los jóvenes que inicialmente se alejan de las formas vernáculas van acomodándose poco a poco a ellas, no sin antes dejar una huella con sus innovaciones, probablemente acogidas más tarde como vernáculas. A este respecto, William Labov, citado por Moreno Fernández (2009, p. 48), dice que hay una división periódica de seis fases para la adquisición del inglés llamado estándar [...] que son las siguientes:

1. adquisición de la gramática básica en la primera infancia

2. adquisición del vernáculo entre los 5 y los 12 años

3. desarrollo de la percepción social entre los 14 y los 15 años

4. desarrollo de la variación estilística a partir de los 14 años aproximadamente

5. mantenimiento de su uso "estándar" coherente en primera etapa adulta

6. adquisición de todos los recursos lingüísticos; se produce en las personas instruidas y especialmente preocupadas por el uso de la lengua.

Las etapas 5 y 6, como se ve, son fases de estandarización debidas a la integración del usuario al mercado de trabajo y a las nuevas condiciones de vida, los deberes sociales y 
familiares que se adquieren, los nuevos roles que se deben adoptar y -lo más importanteel cambio del modo de vida.

Hay otras variables como la etnia y el lugar de procedencia, también muy importantes, pero por el momento sería muy pertinente hacer una serie de disquisiciones acerca de la incidencia de esas variables en la enseñanza de elE. Con este propósito se pueden plantear los siguientes interrogantes: a partir de la existencia de las variantes como algo consustancial a la lengua, el uso de esas variantes en el aprendizaje de ele ¿se puede considerar un error o, por el contrario, deben ser objeto de estudio en función del contexto donde tendrá que desenvolverse el aprendiente?, ¿debe dejarse que el aprendiente experimente la variación y el choque cultural?, ¿se debe enseñar solo la variante estándar?

Desde el punto de vista académico, y teniendo en cuenta que quienes llegan a Colombia de un país extranjero -sobre todo brasileños, estadounidenses, europeos y asiáticos-, generalmente mano de obra calificada, de alguna manera llegan vinculados 0 esperan vincularse al mercado de trabajo calificado, lo más indicado es ofrecer la enseñanza de la variedad estándar porque es la de prestigio abierto que le permite al aprendiente desenvolverse social y lingüísticamente en un contexto de formalidad. A propósito de la llegada de extranjeros a Colombia, El Tiempo, en su página editorial del 24 de septiembre de 2014, comentaba que la llegada de ciudadanos de otras nacionalidades

es algo positivo porque permite fortalecer las redes que aseguran la transmisión de ideas, materia prima de la innovación. Que sean cada vez más los profesionales formados en otros lugares y en otras escuelas que llegan a nuestro sector productivo, así como a la academia, hace que el país esté más cerca de los epicentros donde está la vanguardia del conocimiento, sea cual sea el campo, un aspecto clave para la competitividad.

Si se acepta el postulado de que se debe enseñar, aunque no únicamente la variante estándar, y teniendo en cuenta que todas las variantes tienen distintos niveles de prestigio, es conveniente no someter al aprendiente a ningún riesgo de estigmatización social por la variante que maneje y ubicarlo en un nivel de prestigio a partir del cual pueda derivar otras formas lingüísticas y, por las influencias del contexto, optar por la variante que más se adecúe a sus necesidades comunicativas en el entorno donde se encuentre. Esto quiere decir que la enseñanza de la variedad estándar, homogénea, vital, ha de incluir un contenido gramatical que le permita al aprendiente extrapolar a otros contextos enunciativos, en que se conviva con otras normas sociolingüísticas, las normas gramaticales aprendidas, para lo que debe aplicar explicaciones teórico-gramaticales de fácil manejo. Es de anotar que este conocimiento lingüístico-gramatical debe estar en íntimo contacto con los factores 
extralingüísticos que le permitan al nuevo usuario adaptarse a contextos geográficos y culturales más o menos amplios donde se den otros usos lingüísticos.

Esto implica que la enseñanza de la lengua extranjera debe tener un contenido gramatical explícito de fácil aprendizaje y manejo para que, haciendo uso de su creatividad -en el sentido chomskiano-, el aprendiente pueda hacer aplicaciones y construcciones enunciativas, así no las haya escuchado con anterioridad. Negando esta opción, es posible que se someta al neousuario a ser un mero repetidor de expresiones tipo estímulorespuesta. No se trata, obviamente, de centralizar el trabajo en dictar normas gramaticales o prescripciones del "buen decir", pero sí es necesario adentrarse en la lógica de la lengua y darle al estudiante herramientas teóricas de fácil aprendizaje y manejo que generalicen un hecho gramatical que luego, con el uso, irá acomodando, como podrían ser las formas de masculino y femenino, la formación del plural y la concordancia en género y número entre el sustantivo y el adjetivo, entre otras. De la misma manera, en el caso de que el enseñante tuviera algún conocimiento de la lengua del aprendiente, se podrían utilizar patrones lingüísticos comunes a las dos lenguas para comparar las estructuras, en virtud de ciertos principios universales que tienen las lenguas, lo que le permitiría contrastar los sistemas, predecir estructuras, hacer más fácil el aprendizaje y más normatizado su uso. A este propósito, cabe recordar un concepto fundamental aportado por Montes (1995, p. 27) cuando define la norma linguística como "la convención tradicionalizada, incluida en el sistema de reglas que hacen y mantienen la cohesión dentro de una comunidad humana". Es obvio que se refiere tanto a la corrección gramatical como a la adecuación pragmática.

En este propósito de trabajar la variante normatizada, es importante la utilización de los medios de comunicación, que tienen mucha injerencia en la opinión pública nacional por ser -supuestamente- los que le dan un uso más "prestigioso" a la lengua. A este respecto, Alfaro Logoria (1998) afirma:

Los medios de comunicación, tanto la prensa escrita como los audiovisuales, han pasado a jugar un papel importante en las representaciones del proceso de estandarización al interior de una comunidad linguística. En la medida en que las emisiones globalizantes, tienden a proyectarse para grandes audiencias y/o para un público lector de iguales características, las diferencias dialectales de naturaleza léxica 0 sintáctica tienden a atenuarse.

Utilizar los medios más altamente cualificados desde el punto de vista tecnológico y empresarial tiene la ventaja, además, de familiarizar a los aprendientes con los problemas reales de la nación, su política, su economía, su educación, que sin duda le aportarán un conocimiento, aunque parcial, de la razón de ser de los usos contextuales y en gran 
medida ampliarán su universo cognitivo en cuanto al contexto, lo que lo integrará mejor a la sociedad y a la cultura.

Es necesario insistir en que el conocimiento lingüístico estructural y el uso cultural de la lengua en ningún momento se pueden considerar aislados. Las culturas no son entidades aisladas de su lengua; ambas constituyen una unidad indisoluble y cada una lengua y cultura- exige ser utilizada mancomunadamente en la forma en que la sociedad considere correcta y adecuada. Esta actitud frente a la realidad está en concordancia con la definición de cultura que aporta Goodenough (1957, p. 167) cuando asegura: "La cultura es todo aquello que una persona debe saber o creer para desenvolverse de forma adecuada entre los miembros de un grupo humano concreto y para cumplir una función aceptada por todos ellos. Este conocimiento se adquiere y aprende en un proceso de socialización". Por esta razón, es necesario insistir en la corrección como criterio incluyente en el manejo de la lengua. La pragmática, como ciencia que tiene su fundamento en el manejo de las regulaciones al usar la lengua en eventos concretos, utiliza la adecuación como criterio para referirse a la condición necesaria para el desenvolvimiento integrativo de los miembros de una comunidad y de los participantes en ella, independientemente de su proveniencia 0 cualquier otra variable social.

La cultura es el factor más determinante de las representaciones simbólicas que cada sociedad hace de su realidad, significancias de que dependen las diferentes formas de vida y los comportamientos individuales dentro del contexto de relación elaborada a lo largo de una historia social que construye unos vínculos y una forma específica de manejar la dinámica interaccional dentro del grupo. Así se construyen las culturas, a partir de las diferentes praxis y de los roles individuales organizados para orientar la percepción del mundo, subcategorizarlo y evaluar los comportamientos en una escala cuyos extremos son lo prestigioso y lo estigmatizado.

Un comportamiento lingüístico prestigioso es el que se acomoda a todas las exigencias de la interacción en comunidades de habla concretas y se caracteriza por mantener la comunicación a pesar de las diferencias que se pueden dar entre los interlocutores. Es decir, son todos aquellos comportamientos lingǘsticos o no que se acomodan al contexto de manera tal que integren a los actores de la interacción y creen convergencia entre ellos. Una variedad lingüística estándar crea este tipo de relaciones respetuosas y rompe las divergencias o interferencias que podrían darse como consecuencia de la diversidad cultural o lingüística; generalmente, el usuario de la variante prestigiosa es bien evaluado, goza de aprobación social desde el primer contacto y, en cierta medida, resalta su imagen positiva frente al interlocutor dadas sus muestras de cortesía, como principio fundamental de las interacciones lingüísticas, y su conducta comunicativa integrativa, como consecuencia de 
la necesidad de ganar confianza y proximidad personal con el interlocutor, o instrumental para obtener algún beneficio inmediato o posterior.

Las variantes subestándares o estigmatizadas -y valga la oportunidad para excluir de esta categoría las variantes regionales, que merecen todo el reconocimiento y el respetoson elaboraciones linguísticas que, si bien se acogen a la normatividad gramatical de la lengua, en todos los casos rompen los esquemas de las culturas y de las sociedades donde se hallan inscritas y se constituyen en contracultura; por esta razón, es conveniente ubicar las variantes regionales en otra clasificación. Las variantes regionales son, sin duda, indicios de la diversidad cultural que agrupa a una lengua dentro de una hipotética comunidad lingüística; es decir, una lengua, en cuanto sistema, puede albergar una amplia gama de culturas, cada una de las cuales le da una materialización y un uso en función de las necesidades del entorno físico donde se socializa. Esto se hace claro en las palabras de Halliday (1998, p. 30) cuando asegura que "[1] a lengua es como es por lo que tiene que hacer".

Las variantes regionales de una lengua -que perfectamente se podrían denominar "idiomas" en virtud de la relación etimológica de esta palabra, por ejemplo, con idiosincrasia - son formas particulares que adopta el habla en un contexto determinado y de alguna forma recogen toda su historia, sus tensiones internas, sus diferencias grupales y el sentir de una comunidad socioculturalmente establecida en un territorio donde se aplican las mismas reglas gramaticales que la variante estándar pero se utilizan formas lingüísticas propias -aloformas- para materializar la propia cognición social y la semiótica cultural particular. Estas formas regionales se diferencian de la variante estándar en cuanto tienen ciertos elementos de uso propios de una comunidad que no rompen el sistema y le dan relevancia a lo cultural propio por encima de la generalidad de una nación.

\section{Conclusión}

Es evidente la necesidad de construir espacios en la enseñanza de ELE en que se integren todos los elementos simbólicos que construyen la sociedad y la cultura para que el aprendizaje sea realmente significativo y los aprendientes encuentren contextos reales de interacción donde la gente actúa y establece distintos tipos de relaciones propias del entorno, incluso rompiendo a veces los esquemas seculares del sistema lingüístico, pero que satisfacen las necesidades de comunicación. El aprendiente, sin embargo, debe saber utilizar un nivel de lengua estándar que debe aplicar sobre todo en contextos de formalidad, razón por la cual debe familiarizarse con este uso en una primera fase de aprendizaje. 


\section{Referencias bibliográficas}

Almeida, M. (1999). Sociolingüística. La Laguna: Universidad de La Laguna.

Caicedo H., M. (1996). Diferenciación dialectal en el español hablado en Buenaventura. Cali: Imprenta Departamental del Valle.

"Destino cotizado" [editorial], en El Tiempo, Bogotá, 24 de septiembre, p. 14.

Dressler, W.U. (1986). Forma y función de los interfijos. Revista española de lingüística, 16(2), 381-395.

Goodenough, W. H. (1957). Cultural Anthropology and Linguistic. En P. L. Garvin (ed.) Report of the seventh round table meeting on Linguistics and Languje study. Washington, D. C.: Georgetown University Press, pp. 787-805.

Guy, R. G. (1992). Lenguaje y clase social. En Panorama de la lingüística moderna. Madrid: Gráficas Rógar, pp. 57-86.

Halliday, M. A. K. (1982). El lenguaje como semiótica social. México: Fondo de Cultura Económica.

Hackley, N., \& Puello, S. (s.f.). Formación de profesores de español como lengua extranjera. Modos de conducir el aula. Bogotá: Fundación Universitaria Iberoamericana.

Labov, W. (1966). The Social Stratification of English in New York City. Washington: Center for Applied Linguistics.

Marrugo Silva, N. (2014). "El mandao”, en El Tiempo. Bogotá, 28 de julio, pág. 3. Temas especiales. Suplemento "Cien días de soledad sin Gabo".

Moreno Fernández, F. (2009). Principios de sociolingüística y sociología del lenguaje. Barcelona: Ariel Letras.

Montes Giraldo, J. J. (1995). Dialectología general e hispanoamericana. Bogotá: Instituto Caro y Cuervo.

Patiño Rosselli, C. (2000). Sobre etnolingüística y otros temas. Bogotá: Instituto Caro y Cuervo.

Silva-Corvalán, C. (2001). Sociolingüística y pragmática del español. Washington, D. C.: Georgetown University Press.

Sperber, D., \& Wilson (1986). Loose talk. En S. Davis (ed.) (1991) pp. 540-549.

\section{Cibergrafía}

http//:es-es.facebook.com/Deciloconacentopastuso (agosto 30 de 2014). 